\title{
Inhomogeneous Airy's and Generalized Airy's Equations with Initial and Bounday Conditions
}

\author{
M.H. Hamdan* \\ hamdan@unb.ca * corresponding author \\ Department of Mathematics and Statistics \\ University of New Brunswick, Saint John, N.B., E2L 4L5 \\ Canada \\ S.M. Alzahrani \\ Department of Mathematics, Umm Al-Qura University, Al-Qunfudhah \\ Saudi Arabia \\ M.S. Abu Zaytoon \\ Department of Mathematics, University of Petra, Amman \\ Jordan \\ S. Jayyousi Dajani \\ Department of Mathematics and Computer Science \\ Lake Forest College, Lake Forest, IL 60045 \\ USA
}

Received: March 5, 2021. Received: August 30, 2021. Accepted: September 17, 2021. Published: September $20,2021$.

\begin{abstract}
Inhomogeneous Airy's and Generalized Airy's equations with initial and boundary date are considered in this work. Solutions are obtained for constant and variable forcing functions, and general solutions are expressed in terms of Standard and Generalized Nield-Kuznetsov functions of the first- and second-kinds. Series representations of these functions and their efficient computation methodologies are presented with examples.
\end{abstract}

Keywords - Airy, Generalized, Inhomogeneous, NieldKuznetsov.

\section{INTRODUCTION}

$\mathrm{M}$ any interesting equations of mathematical physics have their roots in the nineteenth century, and arose then due to their various applications in physics. Their solutions have been expressed in the forms of integral functions, which gave rise to various special functions that we know today.

An example of the said equations is Airy's differential equations, which continues to receive considerable attention in the literature $(c f .[1,2,3,4]$ and the references therein) due to its various practical applications and theoretical implications. In addition to its direct applicability in the study of optics, fluid flow and electromagnetism, many other differential equations in mathematical physics can be reduced to Airy's equation by an appropriate change of variables. Its theoretical implications are found in the introduction of other functions that arise when seeking solutions to Airy's equation, and infinite series analysis of their solutions. Many functions are related to, or are expressible in terms of Airy's functions ( $c f$. $[5,6,7]$ and the references therein). Collectively, these functions contribute to the creation of knowledge in their extensions to large arguments and complex domains of validity, [8], and generalizations of these functions whenever possible and necessary, [9].

Computationally, Airy's and other related special functions continue to represent a challenge and give rise to the need for 
efficient computational algorithms, (cf. $[10,11,12]$ and the references therein). In fact, a large number of scientists have devoted the better parts of their careers to provide us with the State-of-the-Art computational methodologies of these special functions ( $c f .[13,14,15,16,17]$ and the references therein).

Although Airy's equation started out in its homogeneous forms, it is now evident that many applications require this equation in its more general, inhomogeneous form with a forcing function that can be either constant of variable. For instance, Airy's inhomogeneous equation proved to be a valuable tool in the analysis of flow over porous layers in the presence of a transition layer, wherein Nield and Kuznetsov [18] showed that Brinkman's equation, which governs the flow in the variable permeability transition layer, can be reduced to Airy's equation. Abu Zaytoon et.al. [19, 20] provided models of variable permeability porous layers that resulted in reducing the governing equations to the generalized Airy's equation.

Applications of the inhomogeneous Airy's equation underscore the fundamental importance of solving initial and boundary value problems, and providing the necessary computational methodologies to evaluate their integral functions. To this end, Nield and Kuznetsov [18] found it convenient to define a new integral function in terms of Airy's functions and their integrals. This provided a methodology of handling the inhomogeneous Airy's equation with a constant forcing function.

In systems theory, Airy's equation and functions arise in connection with Wigner distribution and the establishment of connections between chaotic classical systems and their quantum correspondents ( $c f$. [4] and the references therein). Furthermore, their applications in the theory of elasticity, and in the analysis of Schrodinger equation are well-documented, so are their uses in evaluation of the Franck-Condon factors that arise in connection with the Franck-Condon principle. This principle is used to describe transitions between two electronic states of a molecule, [4]. In cases where variable forcing functions arise in system theory applications, the introduced Nield-Kuznetsov functions will become a viable alternative. At this stage, however, only a few applications that rely on the Nield-Kuznetsov functions have been discussed in the literature.

Properties of this newly introduced integral function have been studied by Hamdan and Kamel, [21, 22], who also introduced an integral function to express the particular solution of Airy's inhomogeneous equation with a variable forcing function. Integral functions suitable for expressing particular solutions of the generalized Airy's equation were introduced by Abu Zaytoon et.al. [19], [20], and Alzahrani et.al. [23]. These functions have been analyzed extensively by the same authors ( $c f .[24,25,6,27,28,29,30,31])$.

The current work stems from our belief that knowledge of equations of mathematical physics, and their associated solutions in the forms of integral and special functions, is an important aspect of modern research. These functions represent our mathematical gems, and their computations are an integral part of our methodologies to expand this knowledge. While it might be true that problems involving Airy's equation or the generalized Airy's equation, with or without a forcing function, can be solved numerically or, in some cases, using power series solutions or even Homotpy Perturbation Methods, there are reasons as to why one needs functions such as Airy's function, Scorer functions, and the Nield-Kuznetsov functions.

These reasons include the fact that knowing the functional form of the solution, its functional components, how the solution depends on these functions, the behaviours of these functions as the parameters change, representations of these functions, their inter-dependence, and the relationships these functions have with other functions, offer us more confidence in our solutions and our robust methodologies. Furthermore, they represent an excellent tool in the transfer of knowledge both inter-discipline and intra-discipline. Suffice it to say that had Sir G.B. Airy obtained an answer to his question rather than a solution in terms of what we now know as Airy's functions, we would be discussing a different topic of interest here.

Connections that Airy's functions and the Nield-Kuznetsov functions have with other functions, such as Bessel functions, offers us further insights into behaviours and methods of evaluation of these functions. Furthermore, extensions of applications of these functions, and the design of methodologies that combine solution methodologies obtained using these functions and other techniques, such as numerical approaches, is quite possible. It will not come as a surprise to us if Airy's functions and the Nield-Kuznetsov functions find their way into grid generation techniques, finite difference methods, or the finite element method. The work of Himeur et.al. [32] discusses, and provides an excellent formulation of a higher order triangular plate finite element using Airy's functions.

While we feel this is just the tip of the iceberg when it comes to advancing knowledge from homogeneous to inhomogeneous equations, and that there are many other equations to consider, our goal here is to provide this most up to date and comprehensive knowledge of four functions that have been developed over the past decade. Two classes of the Nield-Kuznetsov functions are considered (the Standard and the Generalized Nield-Kuznetsov functions), each of which is of two kinds: the first and second kinds. They cover the two cases of inhomogeneities: a constant forcing function (NieldKuznetsov functions of the first kind) and variable forcing functions (Nield-Kuznetsov functions of the second kind). We discuss these functions in connection with initial value and boundary value problems involving both Airy's equation and the generalized Airy's equation. Solutions and their series representations and computational procedures are provided in this work. All computations have been carried out using Maple software package.

\section{PROBLEM FORMULATION}

Consider the following inhomogeneous ordinary differential equations (ODE), known as the generalized Airy's equation, and introduced by Swanson and Headley [9]: 
$\frac{d^{2} y}{d x^{2}}-x^{n} y=f(x)$

where $n$ is a positive integer. If $n=1$, equation (1) reduces to the inhomogeneous Airy's equation, namely:

$\frac{d^{2} y}{d x^{2}}-x y=f(x)$

Initial and boundary conditions associated with equations (1) and (2) are:

Initial Conditions:

$y(0)=\alpha$

$\frac{d y}{d x}(0)=\beta$

where $\alpha$ and $\beta$ are known values.

Boundary Conditions on $\left[a_{1}, a_{2}\right]$ :

$y\left(a_{1}\right)=b_{1}$

$y\left(a_{2}\right)=b_{2}$

where $b_{1}$ and $b_{2}$ are given real numbers.

\section{SOLUTION METHOD}

General solutions to (1) and (2) depend on whether or not the forcing function, $f(x)$, is constant. The following two cases are discussed.

\section{A. Constant Forcing Function}

When the forcing function is a constant, $f(x)=\kappa$, general solutions to (1) and (2) are given, respectively, by:

$y=c_{1 n} A_{n}(x)+c_{2 n} B_{n}(x)-\frac{\pi K}{2 \sqrt{p} \sin (p n)} N_{n}(x)$

$y=c_{1} A_{i}(x)+c_{2} B_{i}(x)-\pi \kappa N_{i}(x)$

where $p=\frac{1}{n+2}$, and $c_{1}, c_{2}, c_{1 n}, c_{2 n}$ are arbitrary constants.

The functions $N_{\mathrm{i}}(x)$ and $N_{n}(x)$, appearing in (7) and (8) are, respectively, the Standard and the Generalized NieldKuznetsov functions of the first-kind. These functions have been discussed in $[21,33]$ and are defined as follows:

$N_{i}(x)=A_{i}(x) \int_{0}^{x} B_{i}(t) d t-B_{i}(x) \int_{0}^{x} A_{i}(t) d t$

$N_{n}(x)=A_{n}(x) \int_{0}^{x} B_{n}(t) d t-B_{n}(x) \int_{0}^{x} A_{n}(t) d t$

First derivatives of (9) and (10) are given, respectively, by

$\frac{d N_{i}(x)}{d x}=\frac{d A_{i}(x)}{d x} \int_{0}^{x} B_{i}(t) d t-\frac{d B_{i}[(x)}{d x} \int_{0}^{x} A_{i}(t) d t$ $\frac{d N_{n}(x)}{d x}=\frac{d A_{n}(x)}{d x} \int_{0}^{x} B_{n}(t) d t-\frac{d B_{n}(x)}{d x} \int_{0}^{x} A_{n}(t) d t$

Equations (9)-(12) give the following values at $x=0$ :

$N_{i}(0)=N_{n}(0)=\frac{d N_{i}(0)}{d x}=\frac{\left.d N_{n}(0)\right)}{d x}=0$

Equations (9)-(12) involve the following functions in their definitions: Airy's functions of the first- and second-kind, $A_{i}(x)$ and $B_{i}(x)$, and the generalized Airy's functions of the first- and second-kind, $A_{n}(x)$ and $B_{n}(x)$. Airy's functions are defined as follows, [4]:

$A_{i}(x)=\frac{1}{\pi} \int_{0}^{\infty} \cos \left(x t+\frac{t^{3}}{a}\right) d t$

$B_{i}(x)=\frac{1}{\pi} \int_{0}^{\infty}\left[\sin \left(x t+\frac{t^{3}}{a}\right)+\exp \left(x t-\frac{t^{3}}{a}\right)\right] d t$

with the non-zero Wronskian of $A_{i}(x)$ and $B_{i}(x)$ given by, [4]:

$w\left(A_{i}(x), B_{i}(x)\right)=A_{i}(x) \frac{d B_{i}[x]}{d x}-B_{i}(x) \frac{d A_{i}[x\}}{d x}=\frac{1}{\pi}$

Values of Airy's functions and their derivatives at $x=0$ are expressed in terms of the gamma function, $\Gamma(\mathrm{o})$, as:

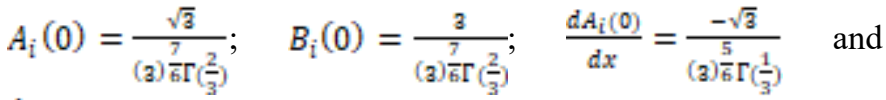

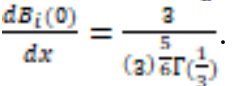

The generalized Airy's functions were introduced by Swanson and Headley, [9], as

$A_{n}(x)=\frac{2 p}{\pi} \sin (p \pi)(x)^{\frac{1}{2}} K_{p}(S)$

$B_{n}(x)=(p x)^{\frac{1}{2}}\left(I_{-p}(S)+I_{p}(\zeta)\right.$

where $I_{p}$ and $K_{p}$ are the modified Bessel functions defined as:

$I_{p}(\zeta)=i^{-p} J_{p}(i \zeta)=\sum_{m=1}^{\infty} \frac{1}{m ! r(m+p+1)}\left(\frac{G}{2}\right)^{2 m+p}$

$K_{p}(\zeta)=\frac{\pi}{2} \frac{\left(L_{-p}(S)-I_{p}(S)\right)}{\sin (p \pi))}$

with $p=\frac{1}{n+2}, \zeta=2 p x$, and $\Gamma($.$) is the gamma function.$

The Wronskian of $A_{n}(x)$ and $B_{n}(x)$ is given by:

$w\left(A_{n}(x), B_{n}(x)\right)=\frac{2}{\pi} p^{\frac{1}{2}} \sin (p \pi)$

and the first derivatives of the generalized Airy's functions are given by, [9]:

$\frac{d A_{n}(x)}{d x}=-\frac{2 p}{\pi} \sin (p \pi)(x)^{\frac{n+1}{2}} K_{p-1}(\zeta)$ 
$\frac{d B_{n}(x)}{d x}=p^{\frac{1}{2}(x)^{\frac{n+1}{2}}}\left[I_{1-p}(\zeta)+I_{p-1}(\zeta)\right]$

When $n=1$ then equation (10) reduces to (9), and the functions $A_{n}(x)$ and $B_{n}(x)$ of (17) and (18) reduce to the functions $A_{i}(x)$ and $B_{i}(x)$ of $(14)$ and (15), respectively. The Wronskian in (21) reduces to the Wronskian in (16).

\section{B. Variable Forcing Function}

When the forcing function is any differentiable function $f(x)$, general solutions to (1) and (2) are given, respectively, by:

$y=c_{1 n} A_{n}(x)+c_{2 n} B_{n}(x)-\frac{\pi}{2 \sqrt{p} \sin (p \pi)} K_{n}(x)$

$y=c_{1} A_{i}(x)+c_{2} B_{i}(x)+\pi K_{i}(x)-\pi f(x) N_{i}(x)$

The functions $K_{\mathrm{i}}(x)$ and $K_{\mathrm{n}}(x)$ are, respectively, the Standard and Generalized Nield-Kuznetsov functions of the second-kind. These functions have been discussed in [21, 33] and are defined as follows, wherein wherein $F^{f} \equiv f$, and prime notation denotes ordinary differentiation:

$K_{i}(x)=f(x) N_{i}(x)-$

$\left\{A_{i}(x) \int_{0}^{x} f(t) B_{i}(t) d t-B_{i}(x) \int_{0}^{x} f(t) A_{i}(t) d t\right\}$

$K_{n}(x)=B_{n}(x) \int_{0}^{x} F(t) \frac{d A_{n}(t)}{d t} d t-A_{n}(x) \int_{0}^{x} F(t) \frac{d B_{n}(t)}{d t} d t$

Equations (26) and (27) give the following first derivatives, respectively:

$$
\begin{aligned}
& \frac{d K_{i}(x)}{d x}=f^{v}(x) N_{i}(x)+f(x) \frac{d N_{i}(x)}{d x}- \\
& \left\{\frac{d A_{i}(x)}{d x} \int_{0}^{x} f(t) B_{i}(t) d t-\frac{d B_{i}(x)}{d x} \int_{0}^{x} f(t) A_{i}(t) d t\right\} \\
& \frac{d K_{n}(x)}{d x}=\frac{d B_{n}(x)}{d x} \int_{0}^{x} F(t) \frac{d A_{n}(t)}{d t} d t- \\
& -\frac{d A_{n}(x)}{d x} \int_{0}^{x^{x}} F(t) \frac{d B_{n}(t)}{d t} d t-\frac{2 \sqrt{p} \sin (p \pi)}{\pi} F(x)
\end{aligned}
$$

\section{COMPUTATIONS OF THE NIELD-KUZNETSOV FUNCTIONS}

In order to solve initial and boundary value problems involving inhomogeneous equations (1) and (2), and to study the behaviour of their solutions, it is imperative to provide computational procedures for their solutions, namely the Nield-Kuznetsov functions of the first- and second-kind. In what follows, we provide the state-of-the-art computational methodologies.

Computing and evaluating $N_{i}(x), K_{i}(x), N_{i}^{t}(x)$ and $K_{i}^{t}(x)$ are based on asymptotic and ascending series representations of Airy's functions. For large values of $x$, we rely on asymptotic series for $A_{i}(x)$ and $B_{i}(x)$, their derivatives and integrals, and for small values of $x$, we use ascending series representations.

\section{A. Asymptotic approximations}

The functions $N_{i}(x), K_{i}(x), \frac{d N_{i}(x)}{d x}$ and $\frac{d K_{i}(x)}{d x}$ can be expressed using the following asymptotic series approximations, given in [12], wherein $\mu=\frac{2}{3} x^{a / 2}$ and $\varphi=\frac{2}{a} t^{2 / a}$ :

$A_{i}(x) \approx \frac{\exp (-\mu)}{2 \sqrt{\pi} x^{4}}\left[1+\frac{(3)(5)}{1 !(-216 j)}+\frac{5(7)(9)(11)}{2 !(-216 j)^{2}}+\cdots\right]$

$B_{i}(x) \approx \frac{\exp (p)}{\sqrt{\pi} x^{\frac{1}{4}}}\left[1+\frac{(8)(5)}{1 !(216 \mu)}+\frac{5(7)(9)(11)}{2 !(216 \mu)^{2}}+\cdots\right]$

$\frac{d A_{i}(x)}{d x} \approx-\frac{x^{\frac{1}{4}} \exp (-\mu)}{2 \sqrt{\pi}}\left[1-\frac{(a)(7)}{1 !(-216 \mu)}-\frac{5(7)(9)(19)}{2 !(-216 \mu)^{2}}+\cdots\right]$

$\frac{d B_{i}(x)}{d x} \approx \frac{x^{\overline{4}} \exp (\mu)}{\sqrt{\pi}}\left[1-\frac{(3)(7)}{1 !(216 \mu)}-\frac{5(7)(9)(13)}{2 !(216 \mu)^{2}}+\cdots\right]$

$\int_{0}^{x} A_{i}(t) d t \approx \frac{1}{a}-\frac{\exp (-p)}{2 \sqrt{\pi} x^{\frac{3}{4}}}\left[1-\frac{41}{48 x^{\frac{2}{2}}}+\frac{9241}{4608 x^{2}}+\cdots\right]$

$\int_{0}^{x} B_{i}(t) d t \approx \frac{\exp (\mu))}{\sqrt{\pi} x^{\frac{3}{4}}}\left[1+\frac{41}{48 x^{\frac{3}{2}}}+\frac{9241}{4608 x^{2}}+\cdots\right]$

Substituting (30)-(35) in (9) and (11), we obtain:

$N_{i}(x) \approx \frac{1}{2 \pi x^{2}}-\frac{1}{a} B_{i}(x)=\frac{1}{2 \pi x^{2}}-\frac{\exp (\mu)}{3 \sqrt{\pi} x^{1 / 4}}$

$\frac{d N_{i}(x)}{d x} \approx-\frac{1}{2 \pi x^{3} f^{2}}-\frac{1}{3} \frac{d B_{i}(x)}{d x}=-\frac{1}{2 \pi x^{3} f^{2}}-\frac{x^{1 / 4} \exp (\mu)}{3 \sqrt{\pi}}$

For large $x$, expressions (36) and (37) take the following forms:

$N_{i}(x) \approx-\frac{1}{3} B_{i}(x)=-\frac{\exp (\mu)}{3 \sqrt{\pi} x^{1 / 4}}$

$\frac{d N_{i}(x)}{d x} \approx-\frac{1}{a} \frac{d B_{i}(x)}{d x}=-\frac{x^{1 / 4} \exp (\mu)}{3 \sqrt{\pi}}$

Similarly, the function $K i(x)$ and its derivative can be approximated asymptotically by using the above approximations in (26) and (28) to get:

$$
\begin{aligned}
& K_{\mathrm{i}}(x) \approx \frac{\exp [-\mu)}{2 \sqrt{\pi} x^{1 / 4}} \int_{0}^{x}\left\{\frac{\exp [\varphi]}{\sqrt{\pi} t^{3 / 4}}\right\} f^{\prime}(t) d t-\frac{\exp (\mu)}{3 \sqrt{\pi} x^{1 / 4}} f(x) . \\
& \frac{d K_{i}(x)}{d x} \approx+\frac{f^{\prime}(t)}{2 \pi x}-\frac{x^{\frac{1}{4}} \exp (-p)}{2 \sqrt{\pi}} \int_{0}^{x}\left\{\frac{\exp (\varphi)}{\sqrt{\pi} t^{\frac{3}{4}}}\right\} f^{y}(t) d t \\
& -\frac{\exp (p)}{3 \sqrt{\pi}}\left\{x^{-\frac{1}{4}} f^{\prime}(x)+x^{\frac{4}{4}} f(x)\right\}
\end{aligned}
$$




\section{B. Ascending Series Representation}

In order to develop ascending series representations for $N_{i}(x), K_{\mathrm{i}}(x), \frac{d N_{i}(x)}{d x}$ and $\frac{d K_{i}(x)}{d x}$, we employ the ascending series representations of the Airy functions, their derivatives and integrals, reported in [12], using Airy's atoms, as follows:

$$
\begin{aligned}
& A_{i}(x)=A_{i}(0) F_{1}^{v}(x)+\frac{d A_{i}[0]}{d x} F_{2}^{\prime}(x) \\
& B_{i}(x)=\sqrt{3} A_{i}(0) F^{v}{ }_{1}(x)-\sqrt{3} \frac{d A_{i}(0)}{d x} F_{2}^{v}(x) \\
& \frac{d A_{i}(x)}{d x}=A_{i}(0) F^{H}{ }_{1}(x)+\frac{\left.d A_{i}(0)\right\}}{d x} F^{H}{ }_{2}(x) \\
& \frac{d B_{i}(x)}{d x}=\sqrt{3} A_{i}(0) F^{n}{ }_{1}(x)-\sqrt{3} \frac{d A_{i}(0)}{d x} F^{n}{ }_{2}(x) \\
& \int_{0}^{x} A_{i}(t) d t=A_{i}(0) F_{1}(x)+\frac{d A_{i}(0)}{d x} F_{2}(x) \\
& \int_{0}^{x} B_{i}(x) d t=\sqrt{3} A_{i}(0) F_{1}(x)-\sqrt{3} \frac{d A_{i}(0)}{d x} F_{2}(x)
\end{aligned}
$$

where

$$
\begin{aligned}
& A_{i}(0)=\frac{1}{a^{2} r^{3} \Gamma_{i}^{2}, j} \\
& \frac{d A_{i}(0)}{d x}=-\frac{1}{\left.a^{1 / 2} \Gamma_{0}^{1} \frac{1}{3}\right)}
\end{aligned}
$$

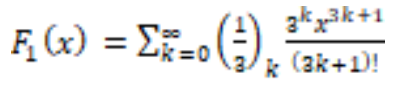

$$
\begin{aligned}
& F_{1}^{t}(x)=\sum_{k=0}^{\mathrm{s}=0}\left(\frac{1}{1}\right)_{k} \frac{\mathrm{a}^{\mathrm{k}} x^{3 k}}{(3 k) !} \\
& F^{\prime \prime}{ }_{1}(x)=\sum_{k=0}^{\mathrm{s}}\left(\frac{1}{3}\right)_{k} \frac{a^{k} x^{3 k-1}}{(a k-1) !} \\
& F_{2}(x)=\sum_{k=0}^{\infty}\left(\frac{2}{2}\right)_{k} \frac{a^{k} x^{3 k+2}}{(3 k+2) !} \\
& F_{2}^{r}(x)=\sum_{k=0}^{\infty}\left(\frac{2}{2}\right)_{k} \frac{a^{k} x^{3 k+1}}{(3 k+1) !} \\
& F_{2}^{\prime \prime}(x)=\sum_{k=0}^{\infty}\left(\frac{2}{a}\right)_{k} \frac{a^{k} x^{3 k}}{(2 k) !}
\end{aligned}
$$

and $(b)_{k}$ is the Pochhammer symbol, defined by, [12]:

$$
(b)_{k}=\frac{\Gamma(b+k)}{\Gamma(b)}=b(b+1)(b+2) \ldots(b+k-1) ;(b)_{0}=1 .
$$

Using (48)-(56) in (9), we obtain:

$$
N i(x)=-2 \sqrt{3} A_{i}(0) \frac{d A_{i}(0)}{d x}\left\{F_{2} F_{1}^{r}-F_{1} F_{2}^{r}\right\}
$$

Differentiating (57), we obtain: $\frac{d N_{i}(x)}{d x}=-2 \sqrt{3} A_{1}(0) \frac{d A_{1}^{\prime}(0)}{d x}\left\{F_{2} F_{1}^{\prime \prime}-F_{1} F_{2}^{n}\right\}$

Upon substituting (50)-(56), the following series are obtained for $N_{i}(x)$ and $\frac{d N_{i}(x)}{d x}$ :

$N_{i}(x)=-2 \sqrt{3} A_{i}(0) \frac{d A_{i}(0)}{d x} *$

$\left[\left\{\sum_{k=0}^{\infty}\left(\frac{1}{9}\right)_{k} \frac{a^{k} x^{3 k}}{(a k) !}\right\}\left\{\sum_{k=0}^{\infty}\left(\frac{2}{a}\right)_{k} \frac{a^{k} x^{3 k+2}}{(a k+2) !}\right\}\right]$

$-2 \sqrt{3} A_{i}(0) \frac{\left.d A_{i}(0)\right)}{d x} *$

$\left[\left\{\sum_{k=0}^{\infty}\left(\frac{2}{3}\right)_{k} \frac{3^{k} x^{3 k+1}}{(3 k+1) !}\right\}\left\{\sum_{k=0}^{\infty}\left(\frac{1}{3}\right)_{k} \frac{3^{k} x^{3 k+1}}{(3 k+1) !}\right\}\right]$

$\frac{d N_{i}(x)}{d x}=-\left[\left\{\sum_{k=0}^{\infty}\left(\frac{1}{2}\right)_{k} \frac{a^{k x^{3 k-1}}}{(2 k-1) !}\right\}\left\{\left\{\sum_{k=0}^{\infty}\left(\frac{2}{2}\right)_{k} \frac{a^{k} x^{3 k+2}}{(2 k+2) !}\right\}\right]\right.$

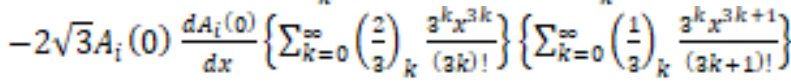

Equations (59) and (60) can be expressed in the following form when we make use of the definition of Cauchy product:

$$
\begin{aligned}
& N_{i}(x)=-2 \sqrt{3} A_{i}(0) \frac{d A_{i}(0)}{d x} * \\
& \sum_{k=0}^{\infty} 3^{k}\left\{\sum_{l=0}^{k}\left(\frac{1}{2}\right)_{1}\left(\frac{2}{a}\right)_{k=1}^{d x}\left(\frac{-3 k+61-1}{(31+1) !(a[k-1)+2)]}\right)\right\} x^{2 k+2} \\
& \frac{d N_{i}(x)}{d x}=-2 \sqrt{3} A_{i}(0) \frac{\left.d A_{i}(0)\right)}{d x} \sum_{k=0}^{\infty} 3^{k}(3 k+2) * \\
& \left\{\sum_{1=0}^{k}\left(\frac{1}{3}\right)_{1}\left(\frac{2}{2}\right)_{k-1}\left(\frac{-d k+61-1}{(31+1) !(2 ! k-1)+2) !}\right)\right\} x^{3 k+1}
\end{aligned}
$$

Ascending series representations of $K i(x)$ and $\frac{d K_{i}(x)}{d x}$ are obtained as follows. Using (42) and (43) we obtain, respectively:

$$
\begin{aligned}
& f(x) A_{i}(x)=A_{i}(0) f(x) F^{v}{ }_{1}(x)+\frac{d A_{i}(0)}{d x} f(x) F_{2}^{r}(x) \\
& f(x) B_{i}(x)=\sqrt{3} A_{i}(0) f(x) F_{1}^{r}(x)-\sqrt{3} \frac{d A_{i}[0\}}{d x} f(x) F_{2}^{r}(x)
\end{aligned}
$$

Integrating (63) and (64), we obtain:

$$
\begin{aligned}
& \int_{0}^{x} f(t) A i(t) d t=A_{1}(0)\left\{f(x) F_{1}(x)-\int_{0}^{x} f^{\prime}(t) F_{1}(t) d t\right\}+ \\
& \frac{d A_{1}(0)}{d x}\left\{f(x) F_{2}(x)-\int_{0}^{x} f^{\prime}(t) F_{2}(t) d t\right\}
\end{aligned}
$$

$\int_{0}^{x} f(t) B i(t) d t=\sqrt{3} A_{i}(0)\left\{f(x) F_{1}(x)-\int_{0}^{x} f^{t}(t) F_{1}(t) d t\right\}-$ $\sqrt{3} \frac{d A_{1}^{\prime}(0)}{d x}\left\{f(x) F_{2}(x)-\int_{0}^{x} f^{\prime}(t) F_{2}(t) d t\right\}$

Using (57), (65) and (66) in equations (26) and (28), respectively, gives: 
$K i(x)=-2 \sqrt{3} A_{i}(0) \frac{d A_{i}(0)}{d x}\left\{F_{1}^{v}(x) \int_{0}^{x} f^{t}(t) F_{2}(t) d t-\right.$ $\left.F_{2}^{r}(x) \int_{0}^{x} f^{t}(t) F_{1}(t) d t\right\}$

$\frac{d k_{i}(x)}{d x}=f^{\prime}(x) N i(x)-$

$2 \sqrt{3} A_{i}(0) \frac{d A_{l}[0)}{d x}\left\{F_{1}^{n}{ }_{1}(x) \int_{0}^{x} f^{s}(t) F_{2}(t) d t-\right.$

$\left.F_{2}{ }_{2}(x) \int_{0}^{x} f^{s}(t) F_{1}(t) d t\right\}$

Upon using (50)-(55) in (67) and (68), we obtain:

$$
\begin{aligned}
& K i(x)=-2 \sqrt{3} A_{i}(0) \frac{d A_{i}(0)}{d x} * \\
& \left\{\begin{array}{c}
\left(\sum_{k=0}^{\infty}\left(\frac{1}{3}\right) \frac{3^{k} x^{3 k}}{(3 k) !}\right) \int_{0}^{x}\left[f^{\left.a(t) \sum_{k=0}^{\infty}\left(\frac{2}{9}\right)_{k} \frac{a^{k} x^{3 k}+2}{(3 k+2) !}\right] d t}\right. \\
-\left(\sum_{k=0}^{\infty}\left(\frac{2}{3}\right)_{k} \frac{3^{k} x^{3 k+1}}{(3 k+1) !}\right) \int_{0}^{x}\left[f^{\prime}(t) \sum_{k=0}^{\infty}\left(\frac{1}{3}\right)_{k} \frac{3^{k} t^{3 k+1}}{(3 k+1) !}\right] d t
\end{array}\right\}
\end{aligned}
$$

$$
\begin{aligned}
& \frac{d k_{i}(x)}{d x}=f^{\prime}(x) N i(x)-2 \sqrt{3} A_{i}(0) \frac{d A_{i}^{\prime}(0)}{d x} * \\
& \left\{\begin{array}{l}
\left(\sum_{k=0}^{\infty}\left(\frac{1}{3}\right)_{k} \frac{3^{k} x^{3 k-1}}{(3 k-1) !}\right) \int_{0}^{x}\left[f^{\prime}(t) \sum_{k=0}^{\infty}\left(\frac{2}{3}\right)_{k} \frac{3^{k} t^{3 k+2}}{(3 k+2) !}\right] d t \\
-\left(\sum_{k=0}^{\infty}\left(\frac{2}{3}\right) \frac{3^{k} x^{3 k}}{(3 k) !}\right) \int_{0}^{x}\left[f^{\prime}(t) \sum_{k=0}^{\infty}\left(\frac{1}{3}\right)_{k} \frac{3^{k} t^{3 k+1}}{(3 k+1) !}\right] d t
\end{array}\right\}
\end{aligned}
$$

For the sake of illustration, graph of $N_{i}(x)$ over the interval $[0,1]$ is shown in Fig. 1(a), and comparison, over the same interval, of the graphs of $N_{i}(x), A_{i}(x)$ and $B_{i}(x)$ is shown in Fig. 1(b). Computations are based on ascending series representations of these functions.

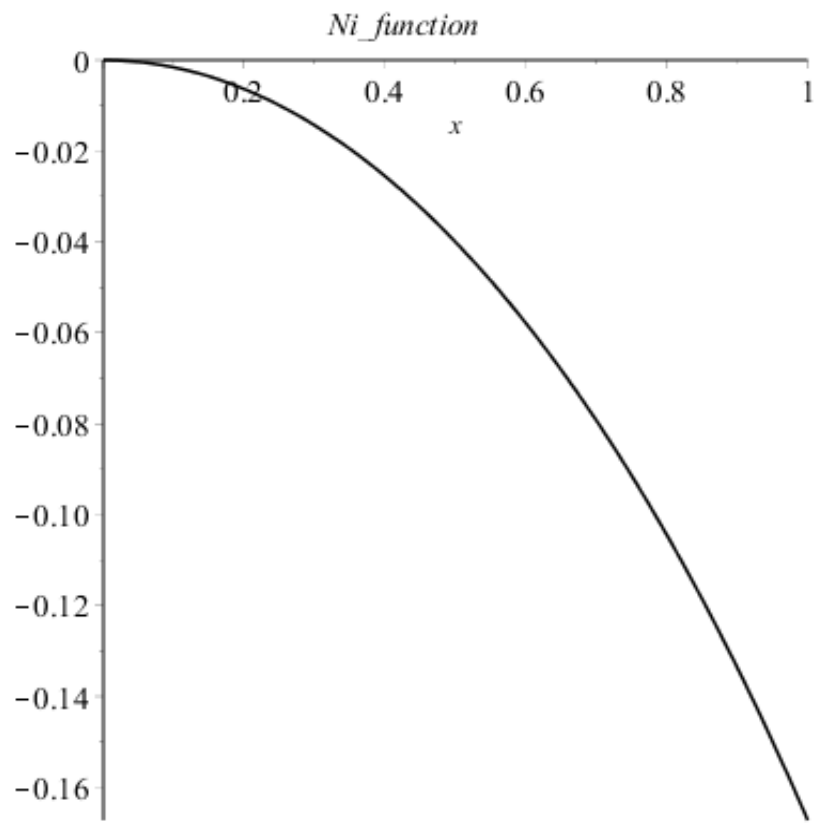

Fig. 1(a) Graph of $N_{i}(x)$ for $0 \leq x \leq 1$

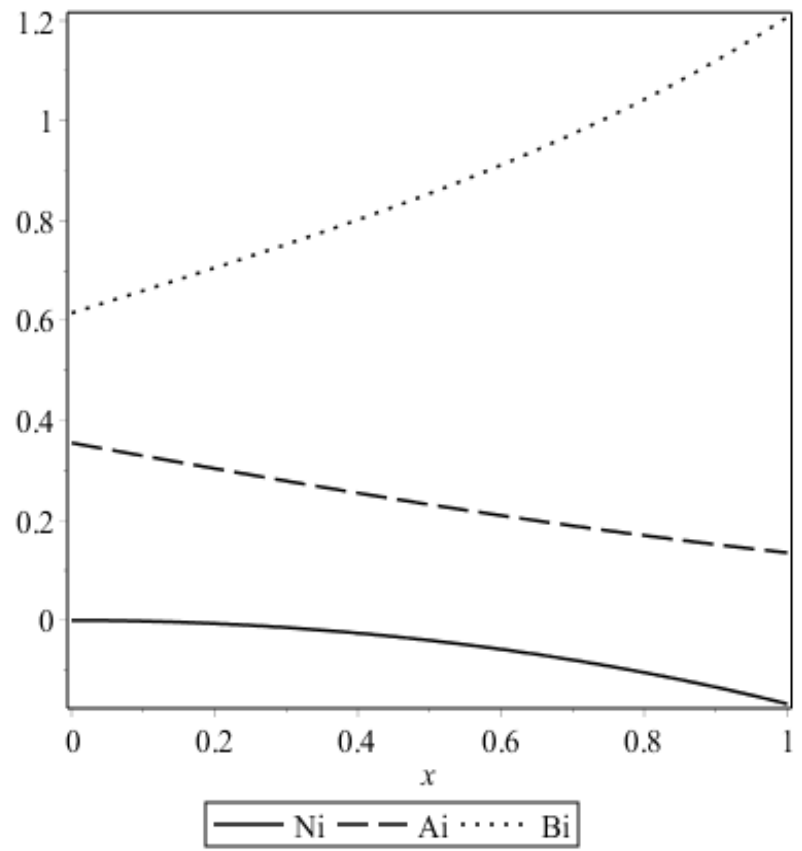

Fig. 1(b) Graphs of $N_{i}(x), A_{i}(x)$ and $B_{i}(x)$ for $0 \leq x \leq 1$

\section{COMPUTATIONS OF THE GENERALIZED NIELD- KUZNETSOV FUNCTIONS}

The generalized Airy's functions have the following series representations, [9], wherein $F^{v}=f$ is the forcing function in equation (1).

Let

$$
\begin{aligned}
& p=\frac{1}{n+2}, \alpha_{n}=\frac{(p)^{1}-p}{\Gamma(1-p)}, \beta_{n}=\frac{(p)^{p}}{\Gamma(p)} \\
& \Theta_{n 1}(x)=1+\sum_{k=1}^{\infty} p^{2 k} \prod_{j=1}^{k} \frac{x^{(n+2 j k}}{j(j-p)} \\
& g_{n 2}(x)=x\left[1+\sum_{k=1}^{\infty} p^{2 k} \prod_{j=1}^{k} \frac{x^{(n+2) k}}{j(j+p)}\right] \\
& g_{n 1}^{\prime}(x)=\sum_{k=1}^{\infty} p^{2 k-1} k^{2}\left(x^{-1+\frac{k^{2}}{p}}\right) \prod_{j=1}^{k} \frac{1}{j(j-p)} \\
& g_{n 2}^{\prime}(x)=1+\sum_{k=1}^{\infty} p^{2 k}\left(1+k^{2} / p\right)\left(x^{\frac{k^{2}}{p}}\right) \prod_{j=1}^{k} \frac{1}{j(j, p)} \\
& \int_{0}^{x} g_{n 1}(t) d t=x+\sum_{k=1}^{\infty} \frac{p^{2 k}}{\left(\left[1+k^{2} / p\right)\right.}\left(x^{1+\frac{k^{2}}{p}}\right) \prod_{j=1}^{k} \frac{1}{j(j-p)}
\end{aligned}
$$


$\int_{0}^{x} g_{n 2}(t) d t=\frac{x^{2}}{2}+\sum_{k=1}^{\infty} \frac{p^{2 k}}{\left(2+k^{2} / p\right)}\left(x^{2+\frac{\mathbb{k}^{2}}{p}}\right) \Pi_{j=1}^{k} \frac{1}{j(j+p)}$

Then

$A_{n}(x)=\alpha_{n} g_{n 1}(x)-\beta_{n} g_{n 2}(x)$

$B_{n}(x)=\frac{1}{\sqrt{p}}\left[\alpha_{n} g_{n 1}(x)+\beta_{n} g_{n 2}(x)\right]$

The generalized Nield-Kuznetsov functions can be evaluated using (72)-(77) in the following expressions:

$N_{n}(x)=\frac{2}{\sqrt{p}} \alpha_{n} \beta_{n}\left\{g_{n 1}(x) \int_{0}^{x} g_{n 2}(t) d t-g_{n 2}(x) \int_{0}^{x} g_{n 1}(t) d t\right\}$

$K_{n}(x)=\frac{-2}{\sqrt{p}} \alpha_{n} \beta_{n}\left\{g_{n 1}(x) \int_{0}^{x} F(t) \frac{d}{d t} g_{n 2}(t) d t-\right.$

$\left.\Theta_{n 2}(x) \int_{0}^{x} F(t) \frac{d}{d t} \vartheta_{n 1}(t) d t\right\}$

$\frac{d N_{n}(x)}{d x}=\left[\alpha_{n} g_{n 1}^{\prime}(x)-\beta_{n} g_{n 2}^{\prime}(x)\right] \int_{0}^{x} \frac{1}{\sqrt{p}}\left[\alpha_{n} g_{n 1}(t)+\right.$

$\left.\beta_{n 2} g_{n 2}(t)\right] d t-\frac{1}{\sqrt{p}}\left[\alpha_{n 1} g_{n 1}^{\prime \prime}(t)+\beta_{n 2} g_{n 2}^{\prime \prime}(t)\right] \int_{0}^{x}\left[\alpha_{n} g_{n 1}(t)-\right.$

$\left.\beta_{n} g_{n 2}(t)\right] d t$

$\frac{d N_{n}(x)}{d x}=\frac{1}{\sqrt{p}}\left[\alpha_{n} g_{n 1}^{\prime}(x)+\beta_{n} g_{n 2}^{\prime}(x)\right] \int_{0}^{x} F(t)\left[\alpha_{n} g_{n 1}^{\prime}(t)-\right.$

$\left.\beta_{n 1} g_{n 2}^{\prime}(t)\right] d t-\left[\alpha_{n} g^{\prime}{ }_{n 1}(x)-\right.$

$\left.\beta_{n 1} g_{n 2}^{\prime}(x)\right] \int_{0}^{x} F(t) \frac{1}{\sqrt{p}}\left[\alpha_{n} g_{n 1}^{\prime}(t)+\beta_{n} g_{n 2}^{\prime}(t)\right] d t-$

$\frac{2 \sqrt{p} \sin (p \pi)}{\pi} F(x)$

The following values at $x=0$ are easily established:

$A_{n}(0)=\frac{(p)^{1-p}}{\Gamma(1-p)} ; B_{n}(0)=\frac{(p)^{1 / 2-p}}{\Gamma(1-p) !} \frac{d A_{n}[(0)}{d x}=-\frac{(p)^{p}}{\Gamma(p))^{p}} ;$

$\frac{d B_{n}(0)}{d x}=-\frac{(p)^{p-1 / 2}}{\Gamma(p)}$;

$N_{n}(0)=0 ; \frac{\left.d N_{n}(0)\right\}}{d x}=0 ; K_{n}(0)=0 ;$

$\frac{\left.d N_{n}(0)\right)}{d x}=-\frac{2 \sqrt{p} \frac{x}{\sin (p \pi)}}{\pi} F(0)$.

\section{SOLUTIONS TO INITIAL AND BOUNDARY VALUE PROBLEMS}

In order to obtain solutions to the initial and boundary value problems, general solutions (7) and (8) must satisfy conditions (3) and (4) for initial value problems, and conditions (5) and (6) for boundary value problems. This requires determination of the arbitrary constants appearing in (7) and (8).

A. Solution to Initial Value Problems (IVP): Constant Forcing Function:
When $f(x)=\kappa$, where $\kappa$ is a given constant, the use of initial conditions (3) and (4) in the general solutions (7), and (8) provides the following expressions for the arbitrary constants $\varepsilon_{1}, c_{2}, c_{1 n}$ and $\varepsilon_{2 n}$ :

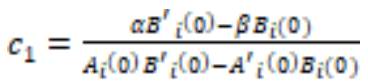

$c_{2}=\frac{-\left[A_{i}^{s}(0)+\beta A_{1}(0)\right.}{A_{i}(0) B_{i}^{r}(0)-A_{i}^{y}(0) B_{i}(0)}$

$c_{1 n}=\frac{\alpha B_{n}^{r}(0)-\beta B_{n}(0)}{A_{n}(0) B_{n}^{r}(0)-A_{n n}^{r}(0) B_{n}(0)}$

$c_{2 n}=\frac{-\alpha A_{n n}^{y}(0)+\beta A_{n}(0)}{A_{n}(0) B_{n n}^{r}(0)-A_{n n}^{r}(0) B_{n}(0)}$

\section{B. Solution to Initial Value Problems (IVP): Variable Forcing Function:}

When $f(x)$ is a differentiable function of $x$, using initial conditions (3) and (4) in the gerenal solutions (7), and (8) yields the following values for the arbitrary constants constants $c_{1}, c_{2}, c_{1 n}$, and $c_{2 n}$ :

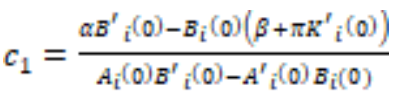

$c_{2}=\frac{-C A_{i}^{y}(0)+A_{i}(0)\left(\beta+\pi X_{i}^{y}(0)\right)}{A_{i}(0) B_{i}^{y}(0)-A_{i}^{y}(0) B_{i}(0)}$

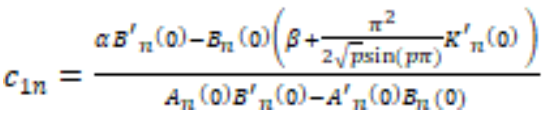

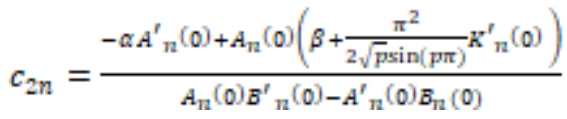

\section{Solution to Boundary Value Problem (BVP): Constant Forcing Function}

When $f(x)=\kappa$, where $\kappa$ is a given constant, using boundary conditions (5) and (6) in the gerenal solutions (7), and (8), we obtain the following values for the arbitrary constants $c_{11}, c_{2}, c_{1 n}$ and $c_{2 n}$ :

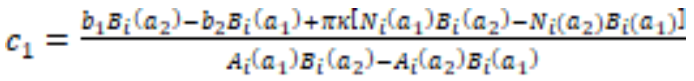

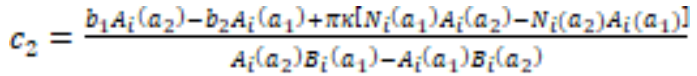

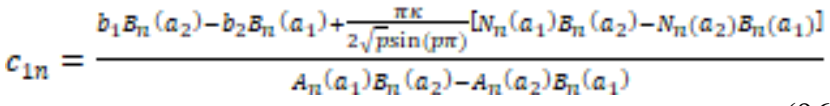

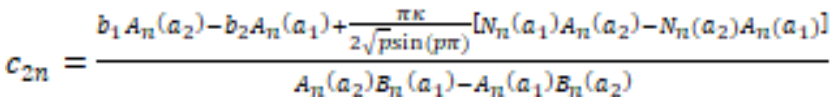




\section{Solution to Boundary Value Problem (BVP): Variable Forcing Function}

When $f(x)$ is a differentiable function of $x$, using boundary conditions (5) and (6) in the gerenal solutions (7) and (8) yields the following expressions for the arbitrary constants $c_{1}, c_{2}, c_{1 n}$ and $c_{2 n}$ :

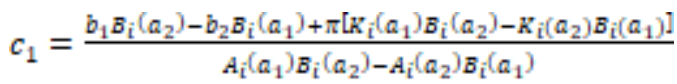

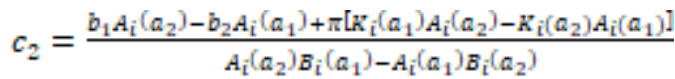

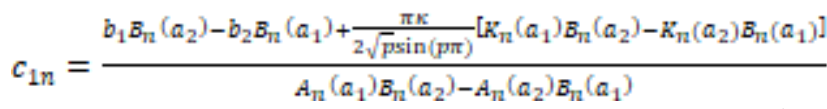

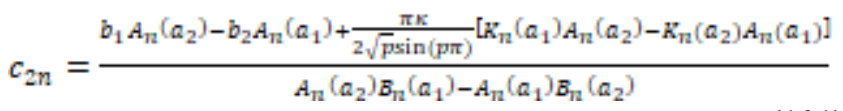

\section{NUMERICAL EXAMPLES}

In order to show numerical values to the arbitrary constants with expressions given by equations (80)-(95), we consider the case of the constant forcing function $f(x)=\mp \frac{1}{\pi}$ and the variable function $f(x)=\sin x$, over the interval $[0,1]$.

\section{A. Initial Value Problem (IVP)}

Considerable attention has been given in the literature to the initial value problem involving Airy's equation (2) subject to conditions (3) and (4), $[6,33]$. We summarize the forms of solutions, in general, for different forcing function $f(x)$ in what follows.

When $f(x)=0$, solution to (2) satisfying (3) and (4) takes the form:

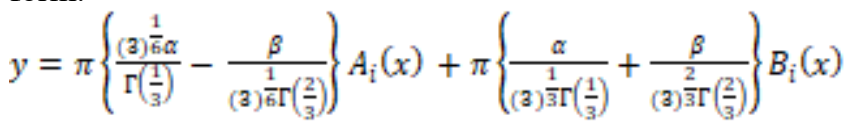

The cases of $f(x)=-\frac{1}{\pi}$, and when $f(x)=\frac{1}{\pi}$ have been considered by Scorer [6] with solutions to the initial value problem given, respectively, by

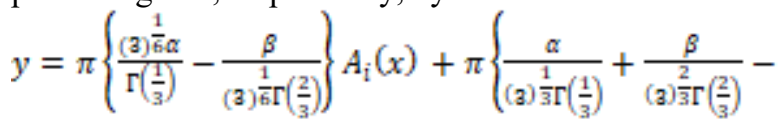
$\left.\frac{1}{3 \Gamma\left(\frac{1}{9}\right) \Gamma\left(\frac{2}{3}\right)}\right\} B_{i}(x)+G_{i}(x)$

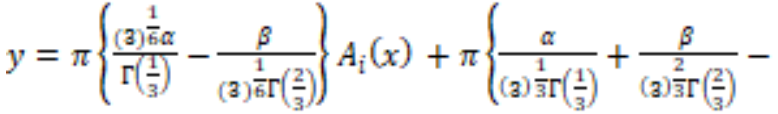

$$
\begin{aligned}
& \left.\frac{4}{(3) \frac{2}{3} \Gamma\left(\frac{1}{3}\right) \Gamma\left(\frac{2}{3}\right)}\right\} B_{\mathrm{i}}(x)+H_{\mathrm{i}}(x)
\end{aligned}
$$

where $G_{i}(x)$ and $H_{i}(x)$ are the Scorer functions, defined by

$$
\begin{aligned}
& G_{i}(x)=\frac{1}{\pi} \int_{0}^{\infty} \sin \left(x t+\frac{t^{3}}{a}\right) d t \\
& H_{i}(x)=\frac{1}{\pi} \int_{0}^{\infty} \exp \left(x t-\frac{t^{3}}{a}\right) d t
\end{aligned}
$$

Values of Scorer functions and their derivatives are given in Table 1, below.

Table 1. Scorer functions and their first derivatives at $x=0$.

\begin{tabular}{|l|}
\hline Integral Function Values at $x=0$ \\
\hline$G i(0)=\frac{1}{3^{7 / 6} \Gamma(2 / 3)}=\frac{A i(0)}{\sqrt{3}}$ \\
$H i(0)=\frac{2}{3^{7 / 6} \Gamma(2 / 3)}=\frac{2}{3} B i(0)$ \\
$\frac{d G i}{d x}(0)=\frac{1}{3^{5 / 6} \Gamma(1 / 3)}=-\frac{1}{\sqrt{3}} \frac{d A i}{d x}(0)$ \\
$\frac{d H i}{d x}(0)=\frac{2}{3^{5 / 6} \Gamma(1 / 3)}=\frac{2}{3} \frac{d B i}{d x}(0)$ \\
\hline
\end{tabular}

When $f(x)$ is a constant function, say $f(x)=x$, Hamdan and Kamel [21] showed that solution to the initial value problem takes the

$$
\begin{aligned}
& y=\pi\left\{\frac{(3)^{\frac{1}{\pi} \alpha}}{\Gamma\left(\frac{1}{3}\right)}-\frac{\beta}{\left.\frac{1}{\left.(a) \frac{(2}{\pi}\right)}\right\} A_{i}(x)+}\right. \\
& \pi\left\{\frac{\alpha}{(3)^{\frac{1}{a}} \Gamma\left(\frac{1}{3}\right)}+\frac{\beta}{(3)^{\frac{2}{2}} \Gamma\left(\frac{2}{3}\right)}-\frac{1}{3}\right\} B_{i}(x)+G_{i}(x)
\end{aligned}
$$

and when $f(x)$ is any differentiable function, solution to the initial value problem is expressible as

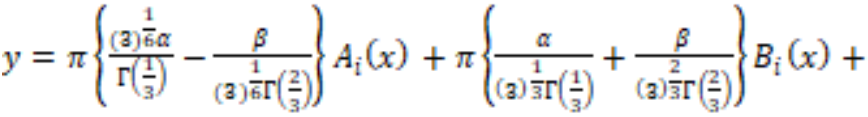

$$
\begin{aligned}
& \pi K_{\mathrm{i}}(x)-\pi f(x) N_{\mathrm{i}}(x)
\end{aligned}
$$

where $N_{i}(x)$ and $K_{\mathrm{f}}(x)$ are given by (9) and (26), respectively.

Comparison of (107) to (103) and (104) shows at the outset that (107) is valid for any $f(x)=\kappa$. In other words, for all chosen $f(x)=\kappa$, values of the arbitrary constants when initial conditions are satisfied, do not change with changes in $f(x)$. 
However, in (103) and (104), values of the arbitrary constants are dependent on $f(x)=\kappa$. This observation also holds for (108) where the arbitrary constants are independent of the (variable) function $f(x)$. It also holds for the generalized Airy's equation (1) subject to conditions (2) and (3).

Solution (107) is equivalent to solutions (103) and (104), for the given values of $K=\frac{1}{\pi}$ and $-\frac{1}{\pi}$ due to the following relationships between the Scorer functions and the standard Nield-Kuznetsov function of the first-kind:

$N_{i}(x)=G_{i}(x)-\frac{1}{a} B_{i}(x)$

$N_{i}(x)=\frac{2}{a} B_{i}(x)-H_{i}(x)$

$N_{i}(x)=\frac{2}{a} G_{i}(x)-\frac{1}{a} H_{i}(x)$

The above observation is validated by computing the values of the arbitrary constants for the case of Airy's equation (2) using expressions (86), (87), (90) and (91), and for generalized Airy's equation (1) using expressions (88), (89), (92) and (93). For Airy's equation (2), values of the arbitrary constants are: $c_{1}=0.884829843$ and $c_{2}=2.74156380$ for all $f(x)$. These have been computed using ascending series representations of the functions involved.

Graphical representations of the solution to the IVP, equation (2) with initial conditions (3) and (4), are illustrated in Fig. 2(a) and 2(b) using computations based on asymptotic and ascending series representations of the functions involved.

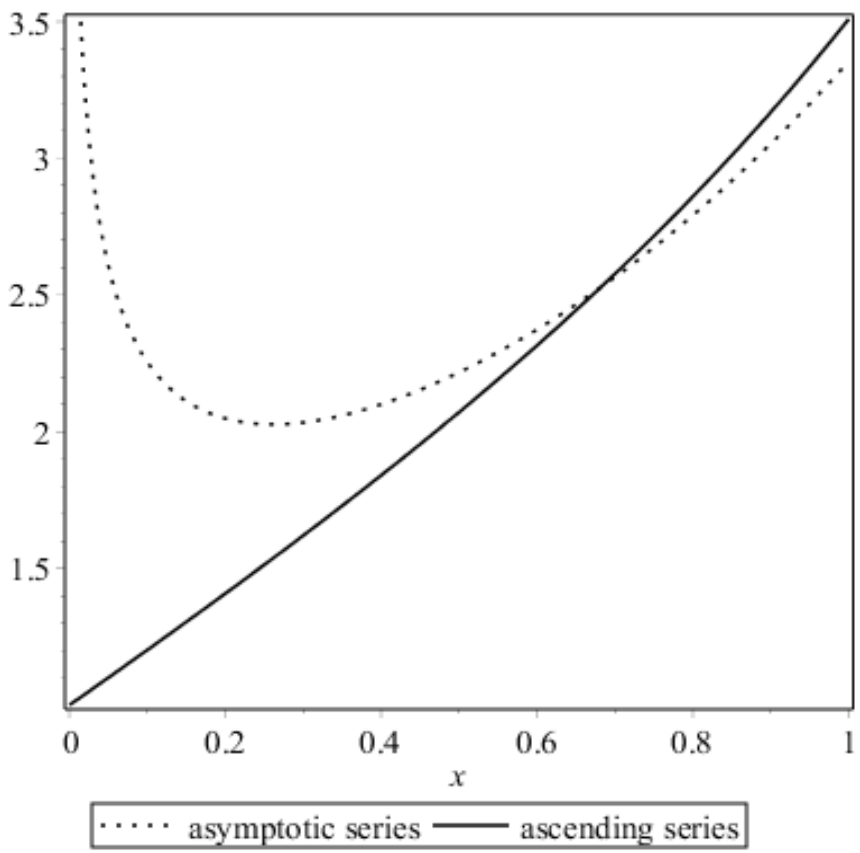

Fig. 2(a): Ascending and Asymptotic Series Solutions:

$$
f(x)=1 / \pi
$$

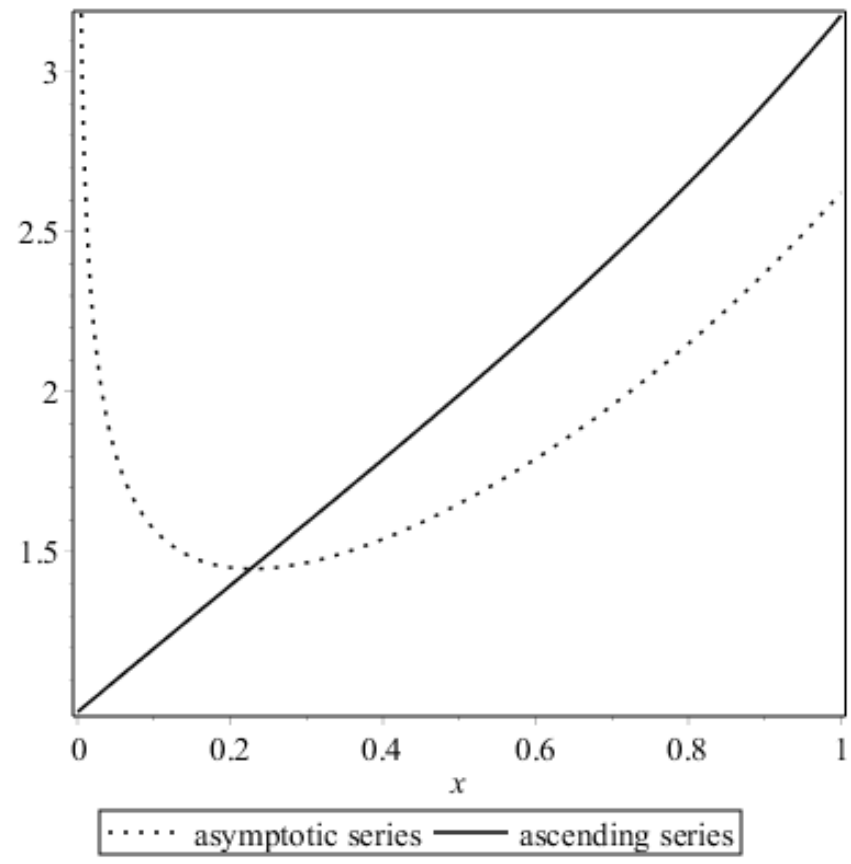

Fig. 2(b): Ascending and Asymptotic Series Solutions:

$$
f(x)=-1 / \pi
$$

For the generalized Airy's equation (1) with initial conditions (3) and (4), values of the arbitrary constants are independent of $f(x)$, but depend on the value of $n$, as shown in Table 2. Arbitrary constants have been computed for $f(x)=\sin x$ and $f(x)=\mp 1 / \pi$.

Table 2. Arbitrary constants for generalized Airy's IVP

\begin{tabular}{|l|l|}
\hline & $\begin{array}{l}f(x) \quad \text { is any forcing } \\
\text { function }\end{array}$ \\
\hline$n=1$ & $\begin{array}{l}c_{1 n}=0.8848298434 \\
c_{2 n}=2.741563801\end{array}$ \\
\hline$n=2$ & $\begin{array}{l}c_{1 n}=0.9023084857 \\
\\
c_{2 n}=3.014847595\end{array}$ \\
\hline$n=5$ & $\begin{array}{l}c_{1 n}=1.538574592 \\
c_{2 n}=3.849602302\end{array}$ \\
\hline$n=10$ & $\begin{array}{c}c_{1 n}=3.224800671 \\
\\
c_{2 n}=5.014277165\end{array}$ \\
\hline
\end{tabular}

\section{B. Boundary Value Problem (BVP)}

Values of the arbitrary constants for the case of Airy's equation (2) subject to boundary conditions (5) and (6) have been computed using expressions (94), (95), (98) and (99). Results are shown in Table 3 and demonstrate the dependence of the values of arbitrary constants on the forcing function $f(x)$.

Table 3. Values of arbitrary constants for Airy's BVP 


\begin{tabular}{|l|l|l|}
\hline$f(x)$ & $c_{1}$ & $c_{2}$ \\
\hline$\frac{1}{\pi}$ & 0.2327830325 & 1.491812929 \\
\hline$-\frac{1}{\pi}$ & -0.362631518 & 1.835575680 \\
\hline $\sin x$ & 0.227012892 & 1.495144321 \\
\hline
\end{tabular}

Values of the arbitrary constants for the case of generalized Airy's equation (1) subject to boundary conditions (5) and (6) have been computed using expressions (96), (97), (100) and (101). Results are shown in Tables 4(a) and 4(b), and demonstrate the dependence of the values of arbitrary constants on the forcing function $f(x)$ and on the value of $n$.

Table 4(a). Arbitrary constants for generalized Airy's BVP

\begin{tabular}{|c|c|c|}
\hline & $f(x)=\frac{1}{\pi}$ & $f(x)=-\frac{1}{\pi}$ \\
\hline$n=1$ & $\begin{array}{l}c_{1 n}=0.2326261442 \\
c_{2 n}=1.491903508\end{array}$ & $\begin{array}{l}c_{1 n}=-0.3624746303 \\
c_{2 n}=1.8354851\end{array}$ \\
\hline$n=2$ & $\begin{array}{l}c_{1 n}=-0.0986091013 \\
c_{2 n}=1.78230547\end{array}$ & $\begin{array}{l}c_{1 n}=-0.901384351 \\
c_{2 n}=2.183693094\end{array}$ \\
\hline$n=5$ & $\begin{array}{l}c_{1 n}=-0.5290973414 \\
c_{2 n}=2.415544415\end{array}$ & $\begin{array}{l}c_{1 n}=-1.899826909 \\
c_{2 n}=2.922621495\end{array}$ \\
\hline$n=10$ & $\begin{array}{l}c_{1 n}=-0.7008235147 \\
c_{2 n}=3.174908784\end{array}$ & $\begin{array}{l}c_{1 n}=-2.950042667 \\
c_{2 n}=3.824202427\end{array}$ \\
\hline
\end{tabular}

Table 4(b). Arbitrary constants for generalized Airy’s BVP

\begin{tabular}{|l|l|}
\hline & $f(x)=\sin x$ \\
\hline$n=1$ & $c_{1 n}=0.2266598861$ \\
& $c_{2 n}=1.495348129$ \\
\hline$n=2$ & $c_{1 n}=-0.1037847989$ \\
& $c_{2 n}=1.784893318$ \\
\hline$n=5$ & $c_{1 n}=-0.5335568124$ \\
& $c_{2 n}=2.417229937$ \\
\hline$n=10$ & $c_{1 n}=-0.7060034923$ \\
& $c_{2 n}=3.176404117$ \\
\hline
\end{tabular}

\section{CONCLUSION}

In this work, we provided analysis of initial and boundary value problems associated with Airy's and generalized Airy's equations. The introduction of the Standard Nield-Kuznetsov functions of the first- and second-kind facilitated obtaining general solutions to the inhomogeneous Airy's equation, while the Generalized Nield-Kuznetsov functions made it possible to find general solutions to the inhomogeneous generalized Airy's equation. This is a novel aspect of this work in which we presented the state-of-the-art computational methodologies for evaluating the Nield-Kuznetsov functions based on series analysis of their constituents, namely Airy's functions.

As many problems in Mathematical Physics are modelled or are reducible to Airy's equation, this work is relevant to research work in areas such as fluid mechanics and systems theory. With the knowledge and methods introduced in this work, one is now able to solve the inhomogeneous Airy's ODE and the inhomogeneous generalized Airy's ODE that involve initial value problems and two-point boundary value problems.

Evaluation of all of the Nield-Kuznetsov functions relies heavily on computer simulation. While Airy's functions and their series representations have built-in definitions in Maple and Mathematica, the Nield-Kuznetsov functions are not presently included. Series representations for the NieldKuznetsov functions are available and can be evaluated using Maple or Mathematica, through their definitions in terms of Airy's functions or in terms of series computations, as has been done in [19] to [31].

\section{References}

[1] M. Abramowitz and I.A. Stegun, Handbook of Mathematical Functions, Dover, New York 1984.

[2] N. M. Temme, "Parabolic Cylinder Functions", in NIST Handbook of Mathematical Functions", Chapter 12, pp. 303-319, (Frank W. J. Olver, Daniel W. Lozier, Ronald F. Boisvert and Charles W. Clark, eds.), U.S. Dept. Commerce, Washington, DC, Cambridge University, 2000.

[3] N.M. Temme. Special functions: An introduction to the classical functions of mathematical physics. John Wiley \& Sons, New York, 1996.

[4] O. Vallée and M. Soares, Airy functions and applications to physics. World Scientific, London, 2004.

[5] J. C. P. Miller and Z. Mursi, Notes on the solution of the equation $y^{x t}-x y=f(x)$, Quarterly J. Mech. Appl. Math., vol. 3, pp. 113-118, 1950.

[6] R.S. Scorer, "Numerical evaluation of integrals of the form $I=J^{-1} f(x) e^{i \varphi(x)} d x$ and the tabulation of the function $=\int_{n} \sin \left(u z+u^{a}\right) d u "$, Quarterly J. Mech. Appl. Math., vol. 3, pp. 107-112, 1950.

[7] S.-Y. Lee, "The inhomogeneous Airy functions, Gi(z) and Hi(z)", J. Chem. Phys. vol. 72, pp. 332-336, 1980.

[8] T.M. Dunster, "Uniform asymptotic expansions for solutions of the parabolic cylinder and Weber equations", J. Classical Analysis, vol. 17, No. 1, pp. 69-107, 2021.

[9] C.A. Swanson and V.B. Headley, "An extension of Airy's equation", SIAM Journal of Applied Mathematics, vol. 15(6), pp. 1400-1412, 1967.

[10] R.M. Corless, D.J. Jeffrey and H. Rasmussen, "Numerical evaluation of Airy functions with complex arguments". J. Comput. Phys. vol. 99, pp. 106-114, 1992.

[11] A.J. MacLeod, Computation of inhomogeneous Airy functions, J. Comput. Appl. Math. vol. 53, pp. 109-116, 1994.

[12] Z. Schulten, R.G. Gordon and D. G. M. Anderson, "A numerical algorithm for the evaluation of Weber parabolic cylinder functions $U(a, x), V(a, x)$, and $W(a,+/-x) ", J$. Comput. Phys. vol. 42(2), pp. 213-217, 1981.

[13] A. Gil, J. Segura, N.M. Temme, On Non-Oscillating Integrals for Computing inhomogeneous Airy Functions, Mathematics of Computation, vol. 70, pp. 1183-1194, 2001.

[14] A.Gil, J. Segura and N.M. Temme, "Fast and a curate computation of the Weber parabolic cylinder function, 
IMA Journal of numerical analysis, vol. 31, pp. 11941216, 2011.

[15] J. Segura and A. Gil, "Parabolic cylinder functions of integer and half- integer orders of non-negative arguments", Computer Physics Communications, vol. 115, pp. 69-86, 1998.

[16] N.M. Temme, Special Functions: An Introduction to the Classical Functions of Mathematical Physics, Wiley, New York, 1996.

[17] N.M. Temme, "Numerical and asymptotic aspects of parabolic cylinder functions", J. Computational and Applied Mathematics, vol. 121, pp. 221-246, 2000.

[18] D.A. Nield and A.V. Kuznetsov, "The effect of a transition layer between a fluid and a porous medium: shear flow in a channel", Transport in Porous Media, vol. 78, pp. 477-487, 2009.

[19] M. S. Abu Zaytoon, T. L. Alderson and M. H. Hamdan, "Flow through a layered porous configuration with generalized variable permeability", Int. J. of Enhanced Res. in Sci., Tech. and Eng., vol. 5(6), pp. 1-21, 2016.

[20] M.S. Abu Zaytoon, T.L. Alderson, and M.H. Hamdan, "Weber's inhomogeneous differential equation with initial and boundary conditions", Int. J. Open Problems Compt. Math., vol. 9(2), pp. 1-12, 2016.

[21] M.H. Hamdan and M.T. Kamel, "On the Ni(x) integral function and its application to the Airy's non homogeneous equation", Applied Math. Comput., vol. 21 No. 17, pp. 7349-7360, 2011.

[22] M.H. Hamdan and M.T. Kamel; "A Note on the asymptotic aeries aolution to Airy's inhomogeneous equation", Int. J. Open Problems in Computer Science and Mathematics, vol. 4 (2), pp. 155-161, 2011.

[23] S.M. Alzahrani, I. Gadoura, and M.H. Hamdan, "Initial and boundary value problems involving the inhomogeneous Weber equation and the Nield-Kuznetsov parametric function", The International Journal of Engineering and Science, vol.6(3), pp.9-24, 2017.

[24] M. S. Abu Zaytoon, T. L. Alderson and M. H. Hamdan, "Flow through layered media with embedded transition porous layer", Int. J. of Enhanced Res. in Sci., Tech. and Eng., vol. 5(4), pp. 9-26, 2016.

[25] M. S. Abu Zaytoon, T. L. Alderson and M. H. Hamdan, "Flow through a variable permeability Brinkman porous core", J. Appl. Mathematics and physics, vol. 4, pp. 766778, 2016.

[26] S.M. Alzahrani, I. Gadoura and M.H. Hamdan, "Ascending series analysis of the transition layer", Journal of Modern Mechanical Engineering and Technology, vol. 3, pp. 7-16, 2016.

[27] S.M. Alzahrani, I. Gadoura and M.H. Hamdan, "Ascending series solution to Airy's inhomogeneous boundary value problem", Int. J. Open Problems Compt. Math., vol. 9(1), pp. 1-11, 2016.

[28] S.M. Alzahrani, I. Gadoura and M.H. Hamdan, "Representations and computation of the NieldKuznetsov integral iunction", ISOR Journal of Applied Physics, vol. 8(3), pp. 55-67, May-June 2016.

[29] S.M. Alzahrani, I. Gadoura, M.H. Hamdan, "NieldKuznetsov functions of the first- and second kind", IOSR Journal of Applied Physics, vol. 8(3), pp. 47-56, May. Jun. 2016.

[30] S.M. Alzahrani, I. Gadoura, M.H. Hamdan, "Tables of values of the Nield- Kuznetsov functions of the first- and second kind", IOSR Journal of Applied Physics, vol 8(4), Issue 4, pp. 56-67, July-August 2016.

[31] S.M. Alzahrani , M.S. Abu Zaytoon , T.L. Alderson, I. Gadoura and M.H. Hamdan, "On the Nield-Kuznetsov integral function and its applications to Airy's inhomogeneous boundary value problem", Journal of Advances in Physics, vol. 11(5), pp. 3265-3274, January 2016.
[32] M. Himeur, H. Guenfoud and M. Guenfoud, "A Higher order triangular plate finite element using Airy's functions", Advances in Mechanical Engineering, vol. 12(11), pp. 1-19, 2020.

[33] M.H. Hamdan, S. Jayyousi Dajani and M.S. Abu Zaytoon, "Recent advances in the Nield-Kuznetsov functions", WSEAS Transactions on Systems, vol. 20, pp. 178-186, July, 2021.

\section{Contribution of individual authors}

All authors participated in literature review and problem formulation.

S. Jayyousi Dajani and M.S. Abu Zaytoon analysed equations and provided solutions.

S.M. Alzahrani checked solutions, carried out the computations and provided graphs.

M.H. Hamdan planned the work, organized and wrote wrote the manuscript.

\section{Sources of funding}

No funding has been received for this work.

\section{Creative Commons Attribution License 4.0 (Attribution 4.0 International, CC BY 4.0)}

This article is published under the terms of the Creative Commons Attribution License 4.0

https://creativecommons.org/licenses/by/4.0/deed.en_US 